\title{
Spatial-Temporal Mitosis Detection in Phase-Contrast Microscopy via Likelihood Map Estimation by 3DCNN
}

\author{
Kazuya Nishimura $^{1}$ and Ryoma Bise ${ }^{1}$
}

\begin{abstract}
Automated mitotic detection in time-lapse phasecontrast microscopy provides us much information for cell behavior analysis, and thus several mitosis detection methods have been proposed. However, these methods still have two problems; 1) they cannot detect multiple mitosis events when there are closely placed. 2) they do not consider the annotation gaps, which may occur since the appearances of mitosis cells are very similar before and after the annotated frame. In this paper, we propose a novel mitosis detection method that can detect multiple mitosis events in a candidate sequence and mitigate the human annotation gap via estimating spatialtemporal likelihood map by 3DCNN. In this training, the loss gradually decreases with the gap size between groundtruth and estimation. This mitigates the annotation gaps. Our method outperformed the compared methods in terms of F1score using challenging dataset that contains the data under four different conditions. Code is publicly available in https : //github.com/naivete5656/MDMLM.
\end{abstract}

\section{INTRODUCTION}

Living cell analysis has an important role in biomedical research such as investigating the effect of a drug and the analysis of cell fate. To visualize living cell, phasecontrast microscopy, which is a non-invasive technique, has been widely used for long term monitoring cell populations in vitro. Unlike other invasive imaging methods such as fluorescent assays, it allows cell observation without cell staining.

The mitosis detection in phase-contrast microscopy provides much information such as the proliferative behavior of the cell population under specific cultured conditions. It is also expected to improve the automated cell tracking methods [1], [2]. The manual analysis is time-consuming, tedious, and prone to human error for a large amount of data. Therefore, the automated mitosis detection method in the phase-contrast image is necessary and it provides us quantitative information on cell proliferation.

The top row in Fig. 1 shows an example process of mitosis. In this process of mitosis, the circularity and pixel value around the mitosis cell becomes high. Then the cell is splitting into two daughter cells, gradually expanding and return to normal cells. Our aim of this study automatically detects the coordinate $x, y$ and frame $t$ of the moment that the two daughter cells first appeared and the boundary of two cells can be observed.

To detect mitosis events, several methods have been proposed. Almost all methods first extract the candidate sequences, and these methods identify the frame of the

\footnotetext{
${ }^{1}$ Department of Advanced Information Technology, Kyushu University, Fukuoka, Japan

kazuya.nishimura@human.ait.kyushu-u.ac.jp
}

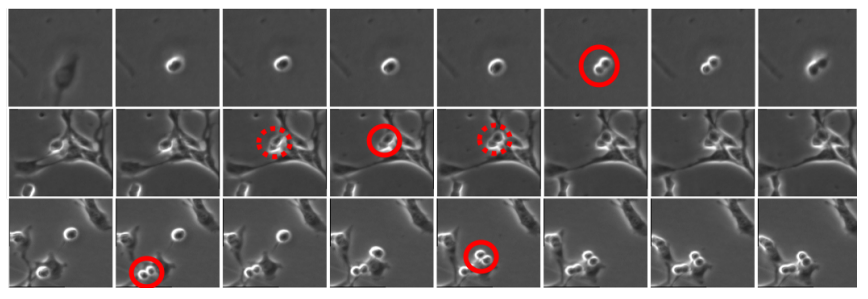

Fig. 1. Example sequences of mitosis process. Red circles indicate human annotations where and when the mitosis event occurs. Top: an easy case that the single cell becomes shrink and bright and then divided into two cells. The timing is clear. Middle: a difficult case that the appearance of the annotated cell (red circle) is similar to those at before and after the frame (red dotted circles). Bottom: a case that multiple mitosis events occur in a near position.

mitosis event from the candidate sequence via graphical model [3], [4], CNN [5], [6], or LSTM [7], [8] to recognize the temporal feature change.

However, these methods still have two problems. First, these methods can not detect multiple mitosis events in a candidate sequence since they assume that a candidate sequence contains only one mitosis event. This situation may occur when the mitosis positions are close as shown in the third row in Fig. 1. In addition, the spatial localization (i.e. mitosis event position) relies on the candidate extraction and it does not use temporal information. The temporal information is only used for temporal localization (i.e. mitosis event timing).

Second, as shown in the red circle and red dotted circle in the middle row in Fig. 1. the appearance of the mitosis cell looks the same before and after the frame. Therefore, the annotations by different annotators may have a temporal gap, and it is difficult for even an expert to annotate with consistent criteria, i.e., it may contain gaps in time. The existing methods estimate a single frame that seems occurs mitosis event from the candidate sequences. If the estimated frame is very close from the ground-truth, the estimate is almost correct and it is different from a miss estimation. Nevertheless, the previous approaches do not consider this situation and it is treated as a miss estimation.

Contribution: The main contribution of this work is to propose a novel mitosis detection method that can detect multiple mitosis events in a candidate sequence and mitigate the human annotation gap. Unlike the previous models that only localize temporal timing, our method can localize the spatio-temporal locations of mitosis events from a candidate sequence. Our model estimates the 3D (2D+t) likelihood map of mitosis events as shown in the network output in 


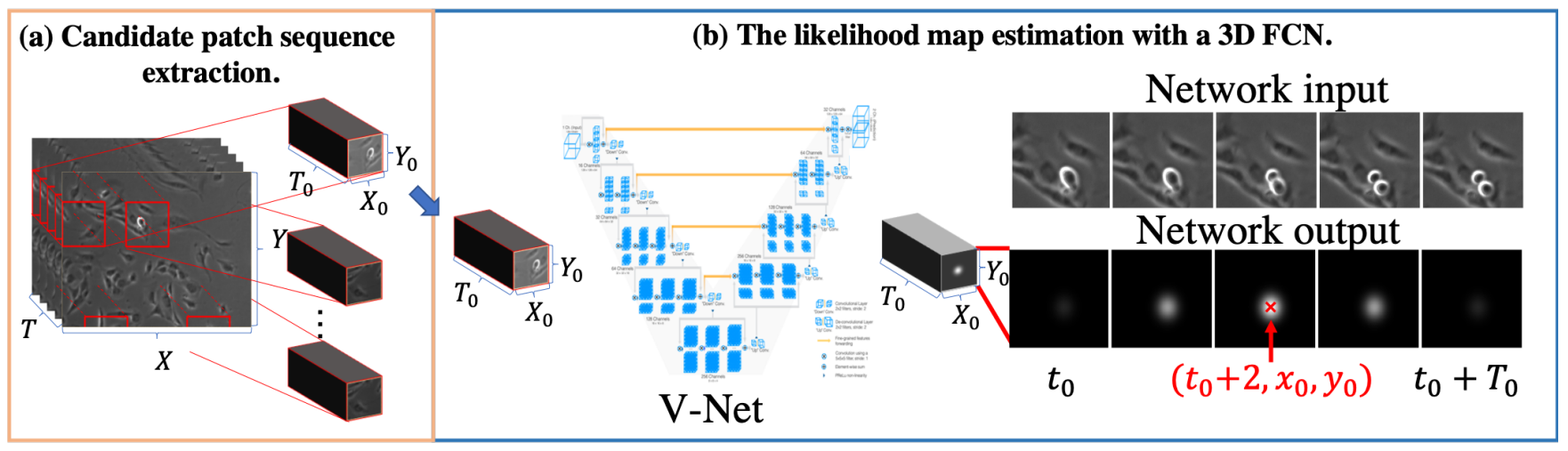

Fig. 2. The overview of the proposed method. (a) First, candidate sequences are extracted based on the fact that the pixel value becomes high in the process of mitosis. b) Then, mitosis events are detected from each candidate via estimating the likelihood map of cell mitosis events by using V-Net [9]. The peak point in the estimated 3D map indicates the mitosis position and timing.

Fig.2. This likelihood map can represent multiple mitosis events in a 3D volume (a candidate sequence), and thus it can detect multiple mitosis events. In addition, we effectively use temporal information for spatial localization by using $3 \mathrm{D}(2 \mathrm{D}+\mathrm{t})$ convolution. Our likelihood map estimation is effective for reducing the affect from the human annotation gap, in which the annotated coordinates become peak with a Gaussian distribution. In the training of our model for estimating the likelihood map, a small gap of spatial-temporal localization gives a small loss, and thus this mitigates the gaps. In experiments, we evaluated our method using a challenging dataset [10] that was used in the CVPR Workshop contest [11] and we outperformed the other compared methods. Our proposed method won second place in the CVPR Workshop contest [11].

Related work: The tracking-based mitosis detection methods first track all cells and then the location of a newly appeared cell in the tracking result is extracted as a candidate for mitosis events. Then, the candidate is classified using visual appearance [2], [12]-[14]. In this approach, mitosis detection relies on cell tracking results. However, the tracking performance significantly decreases when the cell density is high. In addition, the temporal information is not used for classifying the detected candidates if it is true or not. When multiple cells touch each other, the appearance of the touched cells is similar to a mitosis cell. It is difficult to recognize mitosis without temporal information. Therefore, these methods do not achieve enough performance.

As discussed above, the appearance change of a mitosis cell is important information for mitosis detection. To effectively use the temporal context, several graphical modelbased mitosis detection methods that model the change of the visual features in time have been proposed. These methods first extract candidate sequences from the original sequence using image processing. Next, the visual feature of each patch image is extracted using hand-crafted feature extraction such as SIFT [15]. Then the graphical model such as HMM [16] or EDCRF [3] or MGRF [17] MMHCRF+ MM-SMM [18] is used to recognize the mitosis frame from the candidate sequence. These methods achieved better performance than the tracking-based method by benefit from time-series information.

Recently, deep learning is used in the same framework with the graphical model-based method. Nie et al. [5] used 3DCNN for extracting candidate regions and classified by SVM whether the sequence contains mitosis. However, this method does not have the ability to localize when the mitosis occurs in the sequence. Zhou et al. [19] proposed a method that estimates the score map by $2 \mathrm{D} \mathrm{CNN}$, in which the map indicates the candidate of mitosis event on each image by $2 \mathrm{D} \mathrm{CNN}$ and it is classified by $3 \mathrm{D} \mathrm{CNN}$ whether the sequence includes mitosis. However, the input of 3D CNN does not contain cell appearance information, thereby the method can not use the feature extraction ability of 3D CNN. Mao et al. [6], [7] proposed a method that models the appearance changes of a mitosis cell using a bidirectional LSTM for identifying the timing of a mitosis event. Su et al. [8] proposed a model that uses CNN and LSTM for mitosis detection. The CNN extracts the spatial feature and the LSTM extracts the temporal feature. These methods improved the performance compared with those using the hand-crafted feature extraction. However, they assume that a mitosis event occurs one time in the candidate sequence, and thus it is difficult to apply it to the data under the dense condition. In addition, these methods do not deal with the human annotation gap. Sometimes the human may annotate before or after frame that the network estimates. This affects the network training.

To address these problems with multiple mitosis detection and annotation gaps, our method estimates the likelihood map that can represent multiple mitosis events and reduce the bad effects of a human annotation gap.

\section{METHOD}

Fig. 2 shows the overview of our proposed method. Our method consists of following two steps.

a) Candidate patch sequence extraction:

In the entire image sequence, mitosis events appear very sparsely, and thus it occurs the imbalance of true positives and negatives in detection. Learning a $\mathrm{CNN}$ 
with an imbalance dataset takes a lot of time, and it is difficult to converge the training. To mitigate a data imbalance, we first extract candidate sequences that contain the mitosis events by using the intensity-based method based on the fact that the intensity around the cell becomes high when a mitosis event occurs.

b) The likelihood map estimation with a 3D FCN:

Multiple mitosis events may occur in a candidate sequence. Therefore, we handle this problem as a detection problem rather than a classification problem. We detect a mitosis event by estimating the cell mitosis likelihood map for the candidate sequence. To estimate the likelihood map, we use 3D FCN that can estimate each pixel for an input image. In the likelihood map, a mitosis position is represented as an intensity peak with a Gaussian distribution, in which multiple mitosis are represented as multiple peaks.

\section{A. Candidate patch sequence extraction}

We follow the previous work proposed by Huh [3] for candidate patch sequence extraction. Since it is known that the process of mitosis event typically exhibits high brightness, the patch images (i.e. the candidate images that may include some mitosis event.) can be extracted based on the brightness [3]. Each original image is convolved with a small-sized $d \times d$ rectangular average filter. Then the image is binarized by thresholding and the bounding box that has a high probability of including mitosis is obtained from the connected component of the binarized image. Next, the bounding boxes are associated with successive frames based on the distance of the boxes and the candidate sequence is generated (Fig. 2 (a)). We treat the candidate sequence as a $3 \mathrm{D}$ image by considering the time-series as $\mathrm{z}$-slice. It is used the network input. This candidate extraction mitigates the imbalance problem of detection since many regions of false negatives (i.e., non-mitosis regions) are excluded.

\section{B. The likelihood map estimation with a $3 D$ FCN}

To localize the spatial-temporal positions of mitosis events with effectively using the spatial-temporal information, we use a 3D fully convolutional neural network (V-Net [9]), which has been used for 3D segmentation problem. This network can extract the temporal feature by using the z-axis as a temporal axis.

Furthermore, the annotation may be off the accurate cell mitosis timing since the human annotations are not generally strict. To deal with the gap between the human annotation and the accurate cell mitosis position, we use the cell mitosis position likelihood map as training data, where an annotated position becomes a peak and the value gradually decreases with a Gaussian distribution.

The network input is the candidate sequence and the output is the cell mitosis likelihood map which is the same size as the input and the local maxima of output becomes mitosis event position (Fig. 2 (b)).

We generate the cell mitosis likelihood map $L$ to be used as ground-truth at each candidate sequence $i \in N . N$ is the total number of the candidate sequence. To deal with the case that the candidate sequence includes multiple mitosis events, the likelihood map is generated for each annotation in the candidate sequence and these are aggregated by the max operation. Let $\mathbf{p} \in \mathbb{R}^{3}$ denote a location of the likelihood map, and $p_{x}, p_{y}, p_{t}$ are the coordinates of each axis. The likelihood map for the $k$-th annotation $\left(x_{k}, y_{k}, t_{k}\right)$ is defined as,

$L_{i}^{k}(\mathbf{p})=\exp \left(-\left(\frac{\left(p_{x}-x_{k}\right)^{2}}{\sigma_{x}^{2}}+\frac{\left(p_{y}-y_{k}\right)^{2}}{\sigma_{y}^{2}}+\frac{\left(p_{t}-t_{k}\right)^{2}}{\sigma_{t}^{2}}\right)\right)$,

where $\sigma_{x}, \sigma_{y}, \sigma_{z}$ are the hyper parameters which control the spread of a peak.. The likelihood maps $L^{k}(\mathbf{p})(k=1, \ldots, M)$ for each annotation are aggregated by a max operation.

$$
L_{i}(\mathbf{p})=\max _{k}\left(L_{i}^{k}(\mathbf{p})\right)
$$

We use the mean of the squared error loss function (MSE):

$$
\text { Loss }=\frac{1}{N} \sum_{i=1}^{N}\left\|L_{i}-O_{i}\right\|_{2}^{2}
$$

where $O_{i}$ is the network output. We execute a backpropagation algorithm of the computed loss to update the network parameters. In this training, the loss gradually decreases with the gap size between the ground-truth and estimation. It indicates that a small gap gives a small loss in contrast to the previous methods that give the same loss with that of the large gap even for the small gap. This mitigates the annotation gaps.

\section{EXPERIMENTS}

\section{A. Dataset}

We evaluated our method using a challenging open dataset [10] that was used in the CVPR2019 mitosis detection contest [11]. The dataset contains 12 sequences of timelapse images of the myoblast stem cell that were captured by phase-contrast microscopy 1 The images were captured every 5 minutes and each sequence consists of 1013 images with a resolution of $1392 \times 1040$ pixels. The sequences are divided into four groups depending on the growth factor; (a) Control, (b) FGF2, (c) BMP2, (d) FGF2+BMP2 (Fig 3, (a), (b), (c), (d) show the examples of each condition.). There are three sequences for each condition. The human annotation of mitosis events ( $\mathrm{t}, \mathrm{x}, \mathrm{y})$ was given for each sequences. $t$ indicates the time frame, and $x, y$ indicate the spatial coordinate of a mitosis event. In the condition (b) and (d), the appearances of the cells are similar to mitosis cells, i.e., their appearance becomes high intensity and thus it is more difficult to detect mitosis events in these conditions compared with (a), (c).

The V-Net was trained on each cultured condition. We used two sequences as the training data and the other one as the test data. The network input ( $\mathrm{z}$ is a temporal axis) is generated by cropping from the center of the first frame

\footnotetext{
${ }^{1}$ The original dataset contains 12 sequences for training and 4 for the test set. The training data is only opened and the test data is closed. [11]
} 

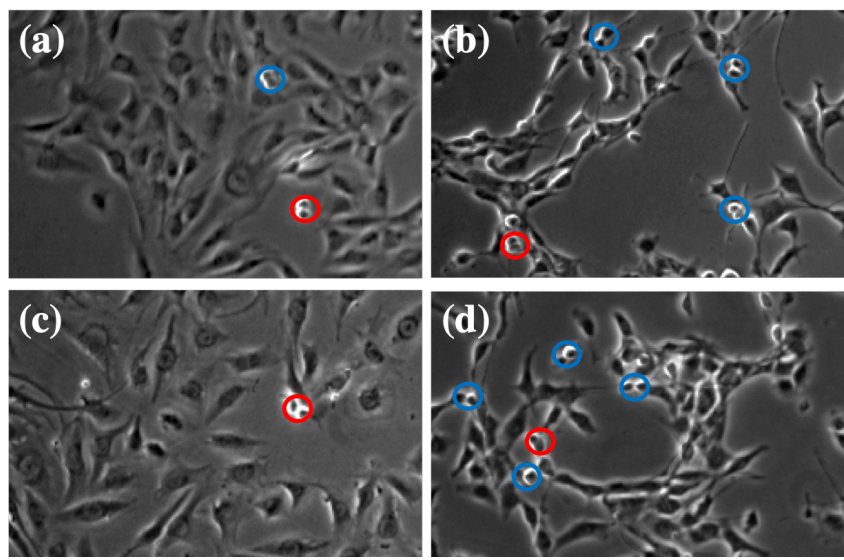

Fig. 3. Example images under various conditions where cells were cultured under growth factors: (a) Control, (b) FGF2, (c) BMP2, (d) FGF2+BMP2 respectively. Red circle indicates a mitosis cell and blue indicates a nonmitosis cell that looks similar to a mitosis event. (b), (d) contain many blue circles hence these are difficult conditions.

of the candidate sequence. The input image size of $\mathrm{V}$-Net is $128 \times 128 \times 16$ in all experiments. If the candidate sequence was shorter than 16 frames, 0 paddings were applied. If the candidate was longer than 16 , the sequence was separated into several candidates by sliding window, where we set the value of the slide as 8 .

\section{B. Detection Accuracy.}

In the experiments, we compared our method with two other methods and one modified our method for ablation study; 1) 3DCNN [5] that classifies whether the candidate sequence contains a mitosis event, 2) EDCRF [3] that uses a CRF to identify the mitosis timing from a candidate sequence. In addition, to show the effect of our regression setup, in which the network produces the likelihood map rather than segmentation, we also evaluted 3) ours w/o cell mitosis likelihood map (CMLM) that uses the same V-Net with the proposed method but it was trained to produce the segmentation results, where the mitosis region is 1 , otherwise 0 . Since 3DCNN [5] does not have temporal localization ability, we set the center coordinate of an input sequence as the timing of the mitosis event.

We used the precision, recall, and F1-score that were used in the contest [11]. If a detected position sufficiently closes to that of a ground-truth (within 6 frames in the temporal direction and 15 pixels in the spatial direction ${ }^{2}$, it is counted as a true positive and if it is far from a ground-truth, it is counted as false positive. If there is no detection points in the local window form a ground-truth, it is counted as a false negative.

The performances of each method are shown in Tab. I] In all conditions, our method outperformed the other methods in terms of F1-score. In addition, compared with Ours w/o CMLM, our method improved in terms of F1-score. This indicates the effectiveness of the proposed likelihood map estimation.

${ }^{2}$ We followed the contest [11] to set these values.

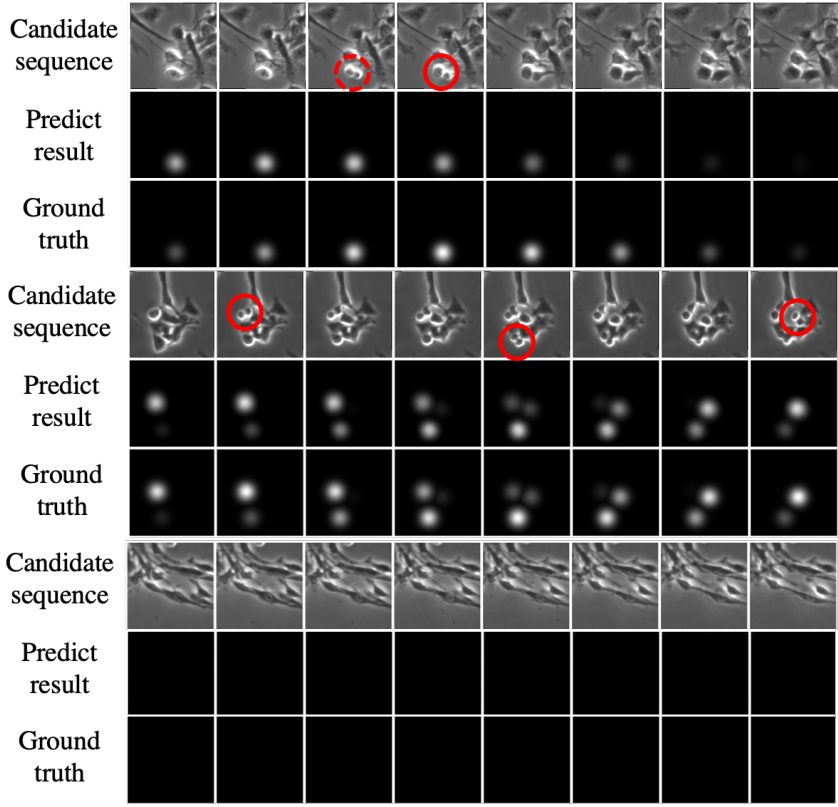

Fig. 4. Examples of mitosis detection results in difficult cases. Top: the sequence that the appearance on the annotated mitosis timing (red circle) is similar to that on the previous frame (dotted red circle). Middle: the sequence that contains multiple mitosis events. Bottom: the negative candidate sequence that contains no mitosis events.

Fig. 4 shows difficult cases due to the similar appearance with the normal cells. The red circle is the annotated position and timing in this examples, and, the dotted circle position also looks like the moment of the mitosis event. As mentioned above, the previous methods treat even one frame gap as a miss estimation in training although the timing may change depending on the annotator. This affects training. On the other hand, in our method, the loss for one frame gap is small since our method estimates the likelihood map as shown in the ground-truth in Fig. 4. Therefore, the adverse effect for training is small and it can achieve robust detection.

In particular, the performance of the compared methods significantly decrease in the condition of FGF2 and FGF2+BMP2, i.e., the difficult cases. In these conditions, the cell density is high where cells shrink and have high intensities, and thus multiple mitosis events often occur in a candidate sequence as shown in Fig. 4. middle. It is difficult for a non-expert to identify all the three mitosis events in the example. In spite of this difficult condition, our method successfully estimated the likelihood map and detected these three mitosis events (Fig. 4). As a result, our method improved the F1-scores over $20 \%$ from those of the other methods in these conditions.

\section{Evaluation of spatial and temporal localization ability.}

In the above experiments, we used the fixed spatialtemporal thresholds to define true positives of detection. In order to show the robustness of our method for the spatial-temporal localization, we evaluated the F1-scores with changing the spatial and temporal thresholds, in which Fig. 5 shows the results for temporal and Fig. 6 for spatial. 
TABLE I

COMPARISON OF MITOSIS DETECTION.

\begin{tabular}{c|c|c|c|c|c|c|c|c|c|c|c|c}
\hline & \multicolumn{3}{|c|}{ Control } & \multicolumn{3}{c|}{ FGF2 } & \multicolumn{3}{c|}{ BMP2 } & \multicolumn{3}{c}{ FGF2+BMP2 } \\
\cline { 2 - 13 } & Precision & Recall & F1 & Precision & Recall & F1 & Precision & Recall & F1 & Precision & Recall & F1 \\
\hline \hline 3DCNN [5] & 0.556 & 0.403 & 0.467 & 0.345 & 0.290 & 0.315 & 0.500 & 0.418 & 0.456 & 0.345 & 0.285 & 0.312 \\
Huh [3] & 0.699 & 0.765 & 0.731 & 0.347 & 0.454 & 0.394 & 0.840 & 0.845 & 0.843 & 0.539 & 0.604 & 0.569 \\
\hline Ours w/o CMLM & 0.813 & 0.865 & 0.838 & 0.345 & $\mathbf{0 . 8 7 5}$ & 0.495 & 0.750 & $\mathbf{0 . 9 7 2}$ & 0.847 & 0.395 & $\mathbf{0 . 8 9 5}$ & 0.548 \\
Ours & $\mathbf{0 . 8 5 7}$ & $\mathbf{0 . 8 9 8}$ & $\mathbf{0 . 8 7 7}$ & $\mathbf{0 . 6 4 6}$ & 0.841 & $\mathbf{0 . 7 3 1}$ & $\mathbf{0 . 8 6 4}$ & 0.947 & $\mathbf{0 . 9 0 4}$ & $\mathbf{0 . 8 3 1}$ & 0.746 & $\mathbf{0 . 7 8 6}$ \\
\hline
\end{tabular}
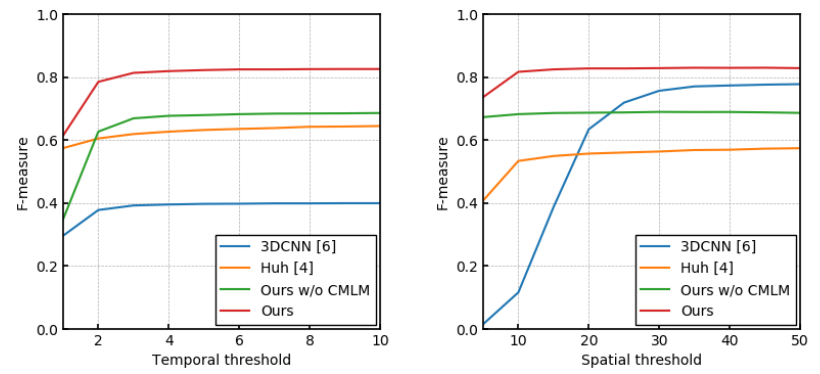

Fig. 5. Comparison of mitosis Fig. 6. Comparison of mitosis event localization on F1-score with event localization on F1-score with changing the temporal thresholds. changing the spatial thresholds.

Here, the high performance with a small threshold indicates the method can accurately localize the spatial position and the timing. In Figs. 6 and 5, the performances of our method were the best at all the thresholds in the comparison. It indicates that our method is effective for both spatial and temporal localization. It is considered for the reason that our method utilizes the spatial-temporal context via 3D convolution for both spatial-temporal localization, in contrast to the other methods that do not use the temporal information for candidate sequence detection (i.e., spatial localization).

\section{CONCLUSION}

In this paper, we proposed an effective method for spatialtemporal mitosis detection by estimating the cell mitosis likelihood map. The method can detect multiple mitosis events and mitigate the annotation gap from ground-truth. In experiments, we confirmed that our proposed method performs better than other methods. In addition, we demonstrated the effectiveness of our method for spatial-temporal localization. The proposed method won second place in the contest of CVPR workshop cell mitosis detection [11].

\section{ACKNOWLEDGMENT}

This work was supported by JSPS KAKENHI Grant Number JP18H05104 and JP19K22895.

\section{REFERENCES}

[1] S. Huh, S. Eom, R. Bise, and et al., "Mitosis detection for stem cell tracking in phase-contrast microscopy images," in ISBI, 2011.

[2] F. Yang, M. A. Mackey, F. Ianzini, and et al., "Cell segmentation, tracking, and mitosis detection using temporal context," in MICCAI, 2005, pp. 302-309.

[3] S. Huh, R. Bise, M. Chen, and et al., "Automated mitosis detection of stem cell populations in phase-contrast microscopy images," IEEE transactions on medical imaging, vol. 30, no. 3, pp. 586-596, 2011.

[4] M. Liu, R. K. Yadav, A. Roy-Chowdhury, and et al., "Automated tracking of stem cell lineages of arabidopsis shoot apex using local graph matching," The Plant Journal, vol. 62, no. 1, pp. 135-147, 2010

[5] W.-Z. Nie, W.-H. Li, A.-A. Liu, and et al., "3d convolutional networksbased mitotic event detection in time-lapse phase contrast microscopy image sequences of stem cell populations," in CVPRW, 2016.

[6] Y. Mao and Z. Yin, "A hierarchical convolutional neural network for mitosis detection in phase-contrast microscopy images," in MICCAI, 2016.

[7] Y. Mao, L. Han, and Z. Yin, "Cell mitosis event analysis in phase contrast microscopy images using deep learning," Medical image analysis, vol. 57, pp. 32-43, 2019.

[8] Y.-T. Su, Y. Lu, M. Chen, and et al., "Spatiotemporal joint mitosis detection using cnn-lstm network in time-lapse phase contrast microscopy images," IEEE Access, vol. 5, pp. 18 033-18 041, 2017.

[9] F. Milletari, N. Navab, and S.-A. Ahmadi, "V-net: Fully convolutional neural networks for volumetric medical image segmentation," in $3 \mathrm{DV}$, 2016, pp. 565-571.

[10] S. Eom, S. Sanami, R. Bise, and et al., "Phase contrast timelapse microscopy datasets with automated and manual cell tracking annotations," Scientific data, vol. 5, p. 180237, 2018.

[11] "CVPR 2019 contest on mitosis detection in phase contrast microscopy image sequences," 2019. [Online]. Available: https: //media.m2i.ac.cn/mitosisdetection/datadescription/

[12] K. Thirusittampalam, M. J. Hossain, O. Ghita, and et al., "A novel framework for cellular tracking and mitosis detection in dense phase contrast microscopy images," IEEE journal of biomedical and health informatics, vol. 17, no. 3, pp. 642-653, 2013.

[13] O. Al-Kofahi, R. J. Radke, S. K. Goderie, and et al., "Automated cell lineage construction: a rapid method to analyze clonal development established with murine neural progenitor cells," Cell cycle, vol. 5, no. 3, pp. 327-335, 2006.

[14] R. Bise, K. Li, S. Eom, and et al., "Reliably tracking partially overlapping neural stem cells in dic microscopy image sequences," in MICCAIW, 2009.

[15] D. G. Lowe, "Distinctive image features from scale-invariant keypoints," International journal of computer vision, vol. 60, no. 2, pp. 91-110, 2004.

[16] G. M. Gallardo, F. Yang, F. Ianzini, and et al., "Mitotic cell recognition with hidden markov models," in Medical Imaging 2004: Visualization, Image-Guided Procedures, and Display, vol. 5367, 2004, pp. 661-668.

[17] A.-A. Liu, J. Tang, W. Nie, and et al., "Multi-grained random fields for mitosis identification in time-lapse phase contrast microscopy image sequences," IEEE transactions on medical imaging, vol. 36, no. 8, pp. 1699-1710, 2017.

[18] A.-A. Liu, K. Li, and T. Kanade, "A semi-markov model for mitosis segmentation in time-lapse phase contrast microscopy image sequences of stem cell populations," IEEE transactions on medical imaging, vol. 31, no. 2, pp. 359-369, 2012.

[19] Y. Zhou, H. Mao, and Z. Yi, "Cell mitosis detection using deep neural networks," Knowledge-Based Systems, vol. 137, pp. 19-28, 2017. 